\title{
AVALIAÇÃo NEUROPSICOLÓGICA ADEQUADA ÀS DEMÊNCIAS
}

\author{
PAULO ROBERTO DE BRITO-MARQUES*, WES JOANETTE**, \\ ARLETTE POISSANT**, BERNADETTE SKA *
}

\begin{abstract}
RESUMO - Os autores discutem aspectos estruturais do Protocole d'Évaluation Neuropsycologique Optimal du Montreal e mostram a possibilidade de sua aplicação no Brasil para a Avaliação Neuropsicológica Adequada à Demências(ANAD). A importância dessa avaliaçăo no exame neuropsicologico das demencias, sobretudo do tipo Alzheimer, é traçar o perfil cognitivo tanto em base transversal como longitudinal. Acreditam que a prática dessa avaliação será de relevância no progresso da pesquisa neurológica, como para esclarecer o espaço virtual entre os limites do envelhecimento normal e patológico.
\end{abstract}

PALAVRAS-CHAVE: Alzheimer, demências, envelhecimento.

\section{Optimal neuropsychological evaluation of dementias}

SUMMARY - The authors discuss the protocol of the Optimal Neuropsychological Evaluation of Dementias of Montreal and its possible application in Brazil. This protocol is important for evaluation in neuropsychological evolution of dementia of the Alzheimer type and for bringing to the fore distinct affectted cognitive profiles as much on a tranversal base as longitudinal. The authors believe that its application would contribute to the progress of research in neurology, as in the clear distinction between normal aging and pathological aging.

KEY WORDS: Alzheimer, dementias, aging.

Na demência o paciente perde as funçōes cognitivas que armazenam o conhecimento humano, a tal ponto que ele chega a não exercer as mais simples tarefas diárias. No começo da síndrome demencial, é difícil diferenciá-la das alterações corticais encontradas no senescente ${ }^{30}$ e seu perfil cognitivo difere entre doenças(doença de Pick e Alzheimer) ${ }^{7}$. A "avaliação neuropsicologica adequada à demência" (ANAD), baseada no Protocole d'Évaluation Neuropsychologique Optimal (PENO), Laboratoire Théophile-Alajouanine, Montréal (Québec) Canada, é instrumento de investigação clínica e de pesquisa que permite verificar tanto componentes (por exemplo: linguagem, memória) quanto subcomponentes (por exemplo, na linguagem: sintático, semântico, fonético) inter e intrafuncionais na organizaçāo do córtex cerebral ${ }^{12}$. A ANAD é limitada pelo seguinte: (1) ela não é capaz de avaliar síndromes demenciais usando conceitos gerais (performance intelectual), porque estes conceitos não estão sedimentados em base cerebral ${ }^{13}$; (2) na doença tipo Alzheimer(DTA) e provavelmente em muitas outras demências, a cognição pode ser afetada de diversas maneiras $e$, por isto, devem ser incluídos na avaliação de uma síndrome demencial todos os componentes e subcomponentes que fazem parte da investigação neuropsicológica ${ }^{13} ;$ e (3) as manifestaçōes neuropsicológicas iniciais de

*Disciplina de Neurologia do Departamento de Medicina Clínica da Faculdade de Ciências Médicas da Universidade de Pernambuco (UPE). Seção de Neurologia do Hospital Oswaldo Cruz da UPE. **Laboratoire Théophile-Alajouanine, Centre de Recherche du Centre hospitalier Côte-des-Neiges, Montréal, Canada. Aceite: 17-agosto-1994.

Dr. Paulo Roberto de Brito Marques - Rua Santa Terezinha 58, Jardim Atlântico - 53140-170 Olinda PE Brasil. 
uma demência não têm sido qualitativamente diferentes das encontradas no idoso normal. Exemplificase este fato com o teste de organização visual de Hooper, que é sensível tanto para o senescente quanto para o demente em fase inicial. Esta falta de poder patognomônico das manifestaçōes neuropsicológicas impede a identificação de marcadores cognitivos qualitativos, quer na demência geral quer em uma doença demencial específica, como a de Alzheimer ${ }^{13}$.

O objeto deste estudo $\hat{e}$ mostrar e discutir o perfil da avaliação neuropsicológica adequada aos casos com suspeita de demência, estendendo-o e dispondo-o à clínica e, principalmente, à pesquisa.

\section{MÉTODO E MATERIAL}

\section{METODO}

É confrontada a sensibilidade da ANAD à do Mini Mental State Examination, introduzido por Folsteins e modificado por Lee Teng e Chang Chuil's. Estes dois últimos testes, de Folstein, não individualizam todos os subcomponentes cognitivos que, de maneira heterogênea, estăo presentes na fase precoce da demência (principalmente por alterą̧öes de linguagem) ${ }^{22}$. A ANAD é constituída de 20 provas que permitem documentar, de modo mais especifico, certos componentes e subcomponentes mais importantes da membria, da linguagerm, das gnosias, das praxias e da atençăo. Estas 20 provas correspondem a um total de 74 itens, em média de 3 a 4 por tarefa. Deve-se efetuar a ANAD em um período de ate 3 horas.

\section{MATERIAL}

ANAD-MEMÓRIA: M1,série de palavras; M2,memória logica; M3,reprodução visual; M4,evocaçăo facilitada e MS, memória a longo prazo.

ANAD-LINGUAGEM: L1,compreensão de palavras; L2,denominação verbal; L3,fluência verbal e semântica; L4, capacidade discursiva; e L5,repetição e leitura de palavras.

ANAD-GNOSIAS: G1,discriminação visual; G2,emparelhamento semântico visual; G3,orientação de linhas; e G4, teste dos Sinos.

ANAD-PRAXIAS: P1,pantomimas; P2, gestos arbitrários; P3, desenho figurativo e geométrico; e P4,cópia da figura de Rey.

ANAD-FUNÇOES EXECUTIVAS: E1,teste de Stroop; e E2,torte de Londres.

\section{RESULTADOS}

Avaliação da memória imediata foi feita por série de palavras, que foi usada no lugar de dígitos, porque: (a) existe forte correlação entre palavra e dígitos seriados; e (b) a série de palavras foi usada como recurso na tarefa M4 (evocação facilitada), ajustando a capacidade de memória a cada pessoa de acordo com a sua própria série. $O$ teste de memória lógica avaliou a capacidade de memória imediata através de material verbal. A cotação foi feita por 5 medidas, que julgam tanto a performance quanto os erros. A prova de reprodução visual também foi tirada da escala clínica de memória de Weschsler. Ela permitiu avaliar capacidades mnésticas usando material não-verbal. A tarefa de evocaçāo facilitada foi item que avaliou a capacidade de evocar uma lista de palavras. A evocação, entretanto, pode ser facilitada insinuando-se tanto a forma quanto a semântica. $O$ item memória a longo prazo permitiu avaliar a capacidade de memória afetada e preservada. Ela conteve 5 subtarefas: (1) evocação livre de palavras, (2) identificaçăo do alvo verbal apresentado entre dois estímulos (visual-fonético e semântico), (3) complementação com silabas sem ligação com os alvos prévios, (4) complementação com sîlabas referindo-se aos alvos prévios, (5) denominação das imagens que correspondem aos alvos verbais.

Avaliação da compreensão de palavras apreciou a capacidade do paciente de emparelhar uma palavra no quadro correspondente. $O$ teste denominação verbal consistiu em avaliar a capacidade do paciente de denominar um objeto num quadro. A prova de fluência verbal e semântica instruiu o paciente a falar num período de 90 segundos o maior número de palavras, respeitando dada categoria 
formal (letra T) ou semântica (nomes de vegetais), todas constituídas de palavras conhecidas (porque, às vezes, 0 acesso ao léxico pode ser afetado, dependendo do critério utilizado na distinção das palavras escolhidas) ${ }^{10}$. Capacidade discursiva $\boldsymbol{E}$ tarefa que avaliou o discurso narrativo, quer previamente conhecido ou graficamente expressado, sobre uma série de desenhos e no discurso de um procedimento (quais são as diferentes etapas necessárias para se fazer um sandwich). $O$ teste de repetição e leitura de palavras avaliou a capacidade fonética: os pacientes repetiram 30 palavras entre as quais 11 foram de uma classe aberta ilimitada, 9 foram de uma classe aberta limitada, 5 foram de uma classe fechada muito frequente e 5 foram não-palavras. Os pacientes também leram em voz alta 30 palavras, entre as quais 12 foram de uma classe aberta ilimitada, 8 foram de uma classe fechada limitada, 5 foram de uma classe fechada muito frequente e 5 foram não-palavras.

Avaliando o domínio gnosia, discriminação visual foi tarefa que apreciou as habilidades visuais básicas não-semanticas. Emparelhamento semantico visual foi prova que avaliou a capacidade do paciente em atribuir um valor semântico a um objeto visualmente percebido (habilidades visuais gnósicas), pedindo-se ao paciente para emparelhar no quadro um alvo que tenha uma funçăo ou categoria relacionadas, em contex to no qual existiam alguns estímulos não associados. Orientação de linhas $\varepsilon$ tarefa tirada de Benton ${ }^{2,3}$, que permitiu analisar algumas habilidades visuo-espaciais básicas. Teste dos Sinos é tarefa em que se pediu ao paciente para localizar um alvo, sistemático e aparentemente casual, distribuido entre grande número de distraçōes.

$\mathrm{Na}$ avaliação da praxia pantomimas uni e bimanuais foram realizadas por pacientes sob comando verbal utilizando tarefas da vida diária (bebendo um copo com água, usando um martelo). Gestos arbitrários foi tarefa que avaliou a habilidade dos pacientes na reproduçăo de gestos năo semânticos, arbitrários e desconhecidos (coloque a mão direita sobre a orelha esquerda). Desenho figurativo e geométrico foi prova em que se pediu ao paciente para realizar 4 desenhos sob comando verbal, 3 figuras geométricas e um outro tipo de figura. Cópia da figura de Rey: nesta tarefa pediuse ao paciente para reproduzir um desenho complexo, aplicando-lhe um escore de acordo com a sua produçăo's.

Avaliando as funções executivas, o teste de Stroop permitiu apreciar: (a) capacidade para nomear cores; (b) habilidade para ler palavras; $e$ (c) capacidade para dizer nomes de cores escritas em cores diferentes, concomitantemente inibindo a atividade da leitura destas palavras com o tipo de cor escrito. No teste da Torre de Londres pediu-se ao paciente para posicionar-se, segundo padrão especifico, diante das bolas de madeira sobre bastões e utilizar número mínimo de tentativas para formar, assim, modelo semelhante ao padrão.

\section{COMENTÁRIOS}

A avaliação da memória é baseada no uso de 5 tarefas que medem: memória imediata; memória episódica de componentes verbal e não verbal; e a extensāo dos itens de memória indisponíveis, a que podem ter acesso quando evocados apropriadamente.

As capacidades linguísticas foram vistas em 5 diferentes tarefas complementares. Estas 5 tarefas permitiram cobertura mínima de 5 componentes diferentes da linguagem, tanto em seus aspectos expressivos quanto receptivos, bem como atender diferentes niveis de componentes, utilizando o nivel de palavras versus o nível de texto. De acordo com alguns autores ${ }^{17}$, a compreensão de palavras pode estar afetada em pacientes dementes, enquanto a denominação verbal e a fluencia verbal e semântica são tarefas mais frequentemente usadas na avaliação da linguagem de pacientes dementes. Entretanto, a capacidade discursiva é um dos aspectos da linguagem raramente visto em pacientes dementes, apesar de ser esta tarefa uma das mais afetadas no exame neurolinguístico de um texto ou mesmo durante o próprio discurso do paciente'. Através do protocolo de afasia MT. $86^{19}$, consegue-se avaliar clinicamente a capacidade fonética do paciente desde os limites mais imprecisos entre a normalidade e uma DTA. Entretanto, todos os pacientes com DTA apresentam, 
no início da doença, déficits de linguagem ${ }^{4}$. Segundo Ska e col. ${ }^{26}$, na maioria dos casos de DTA, as primeiras alterações decorrem de falta de habilidade sintática, seguida pela incapacidade de planejar e organizar os sons da linguagem fonética. Em estudos realizados por Joanette e col. ${ }^{1 \text {, }}$ sobre subcomponentes de diferentes domínios em estágio precoce de pacientes com DTA, foram encontradas diversidades contrastantes na maioria dos pacientes, como por exemplo: déficit de linguagem, com habilidades perceptivas e com integridade lingüística. Mesmo no estágio precoce de pacientes com síndrome demencial, existe riqueza na heterogeneidade das manifestações neuropsicológicas ${ }^{12}$. Por haver lesão precisamente localizada no hemisfério cerebral esquerdo, por exemplo, ela poderá resultar em distintas formas de afasia ${ }^{20}$, tanto que podem afetar mais o plano dos sons das palavras (fonética) como outras, que podem prejudicar mais a organização das sentenças(sintaxe). Esta variedade afasiológica mostra que a linguagem não é somente simples entidade cognitiva, mas que ela necessita de avaliaçăo mais cuidadosa. Mesmo assim, as manifestações neuropsicologicas encontradas na DTA não se apresentam da mesma maneira em todos os pacientes ${ }^{16,18}$. São atividades complexas tanto a organização cerebral da linguagem, como a de outras funções cognitivas. Estas atividades complexas da cognição e organização funcional cortical cerebral também contribuem para muitos outros componentes diferentes (por exemplo: atenção) ${ }^{13}$.

Frequentemente, na avaliação de pacientes dementes (o teste de orientação de linhas é particularmente sensível em pacientes dementes ${ }^{27}$ ) não se incluem as habilidades gnósicas, chamadas capacidades perceptivas. Elas foram introduzidas nesta avaliação, não somente por sua importância na vida diária, mas porque elas podem interferir com outros aspectos de cognição (o teste dos sinos é mais sensível para avaliar atenção espacial que o clássico teste de Albert) ${ }^{31}$. Em alguns casos, uma agnosia subestimada pode confundir-se com um problema de linguagem. Por exemplo, se aplicaçōes gnósicas não forem realizadas, a falta da nomeação de objetos pela tarefa denominativa pode ser confundida com distúrbio de linguagem, ao passo que a verdadeira alteração poderá estar na dificuldade da identificação do quadro representado.

O termo praxia, aqui, inclui tanto os gestos como as praxias visuo-construtivas. Então, o paciente é submetido a duas tarefas que pretendem avaliar suas capacidades gestuais. Estes gestos, em estudos prévios, mostraram ser muito sensiveis em pacientes com demência', ${ }^{1,6}$. A utilização de gestos não aprendidos, que proporcionam informaçōes sobre capacidades gestuais, e duas outras, permite avaliar suas habilidades visuo-construtivas ou gráficas. É sugerido um escore para esta tarefa ${ }^{25}$.

As funçōes executivas têm como objetivo avaliar os distúrbios que representam o protótipo da síndrome do lobo frontal ${ }^{29}$. Duas tarefas foram recentemente adicionadas a respeito: 0 teste de Stroop ${ }^{28}$, ou teste das palavras coloridas, que é baseado em modelo de interferência para permitir a investigação dos componentes do processo de produção verbal e de inibição da resposta; $\mathrm{e}$ a torre de Londres $^{23}$, que aprecia estratégias e planos.

Em conclusão, é necessário chamar a atenção para o que segue.

As diferentes tarefas descritas não precisam ser realizadas na mesma ordem que foram aqui apresentadas. Tanto as tarefas, como partes delas, foram apresentadas de modo pseudopadronizado, relacionado possivelmente à influência de uma tarefa, ou parte dela, sobre outra. Esta avaliação não é perfeita. Entretanto, a maioria dos componentes mais importantes de cognição foi descrita.

Destarte, o princípio fundamental da ANAD, mais do que ela própria, tem que ser enfatizado. O primeiro aspecto a ser acentuado é o de que nossas concepções sobre avaliação detalhada e específica de habilidades neuropsicológicas em manifestações clínicas das demências tem-se confirmado. Se isto não for feito e se apenas procedimentos gerais forem avaliados, esta nossa concepção permaneceria superficial. Por exemplo, existe relação direta entre o conceito das características neuropsicológicas encontradas em pacientes que pertencem a subgrupos clínicos da DTA e o nível refinado de perícia 
e conhecimento para alcançar esta concepção. Acredita-se que somente com a ajuda de procedimento ANAD-like completo seja possível oferecer alguma especificidade na caracterizaçāo de pacientes dementes.

Outra razão para confiar na ANAD é o fato de que a caracterização neuropsicológica de uma demência, ou de um determinado subgrupo clínico de demência, não depende de modelo neuropsicológico (até um certo tempo da evolução). Depende do modelo de sua própria deterioração, a qual será identificada de um modo relativo e refinado utilizando-se todos os aspectos da cognição. Para reconhecer tais trabalhos de pesquisa e de clínica, um procedimento de avaliação como ANAD deverá ser usado.

Finalmente, menciona-se o aumento dos custos administrativos associados à aplicação completa desta avaliação ANAD. Entretanto, é impossível avaliar-se adequadamente aspectos neuropsicologicos de demências com rapidez e material incompleto. Sabe-se que são necessários os mínimos, porém essenciais, procedimentos para uma avaliação requintada. $O$ valor deste correto diagnóstico será de grande importância para especificar um subgrupo clínico em um quadro demencial precoce. E, concomitantemente, é necessário considerar a riqueza de informação que esta avaliação fomecerá no traçado do perfil cognitivo da organização funcional cortical, tanto em base transversal como longitudinal.

\section{REFERENCIAS}

1. Albert ML. A simple test of visual neglect. Neurology 1973, 23:658-664.

2. Benton AL, Hamsher K, Varney NR, Spreen O. Judgement of line orientation. In Contributions to neuropsychological assessment: a clinical manual. New York: Oxford Univ Press, 1983, p 44-54.

3. Benton AL, Varney NR, Hamsher K. Visuospatial judgement: a clinical test. Arch Neurol 1978, 35: 364-367.

4. Cardebat D, Démonet JF, Puel M, Nespoulous JL, Rascol A. Langage et démences. In Habib M, Joanette Y, Puel M. Démences et syndromes démentiels: approche neuropsychologique Paris: Masson \& Edisen, 1991, p 153-164.

5. Folstein MF, Folstein SE, McHugh PR. Mini mental state: a pratical method for grading the cognitive state of patients for the clinician. Psychiat Res 1975, 12: 189-198.

6. Gauthier L, Dehaut F, Joanette Y. The bells test: a quantitative and qualitative test for visual neglect. Internat Neuropsychol 1989, 2: 49-54.

7. Habib M, Joanette Y, Puel M. Démences et syndromes démentiels: approche neuropsychologique. Paris: Masson \& Edissen, 1991.

8. Hooper HE. The Hooper visual organization test manual Los Angeles: Western Psychological Services, 1958.

9. Joanette Y, Brownell HH. Discourse ability and brain damage: theoretical and empirical perspectives. New York: Springer-Verlag, 1990.

10. Joanette $Y$, Goulet $P$. Criterion-specific reduction of verbal fluency in right brain-damaged right-handers. Neuropsychologia 1986, 24: 875-879.

11. Joanette Y, Poissant A, Valdois S. Neuropsychological dissociations in dementia of the Alzheimer type: a multiple single case study. Clin Exp Neuropsychol 1989, 11: 91.

12. Joanette Y, Ska B, Poissant A, Béland R. Neuropsychological aspects of Alzheimer's disease: evidence for inter and infra-function heterogeneity. In Boller F, Khachaturian Z, Poncet M, Christien Y. Heterogeneity of Alzheimer's disease. New York: Springer-Verlag, 1992, p 33-42.

13. Joanette Y, Ska B, Poissant A, Belleville S, Lecours AR, Peretz I. The neuropsychological assessment in studies on dementia: the optimal approach. In Del Ser T,Peña J (eds). Neuropsychological and functional assessment of dementia Barcelona:Masson, 1993

14. Koss E, Ober BA, Dellis DC, Friedland RP. The stroop color-word test: indicator of dementia severity. Internat Neurosci 1984, 24: 53-61.

15. Lee Teng E, Chang Chui H. The modified mini-mental state examination. J Clin Psychiatry 1987 , 48: 314-318.

16. Martin A, Lalonde F, Brouwers P, Cox C, Teleska P, Fedio P, Foster NL, Chase TN. Toward a behavioral typology of Alzheimer's patients. J Clin Exp Neuropsychol 1986, 8: 594-610.

17. Martin A, Fedio P. Word production and comprehension in Alzheimer's disease: the breakdown of semantic knowledge. Brain and Language 1983, 19: 124-141. 
18. Neary D, Snowden JS , Bowen DM, Sims NR Mann D, Benton JS, Northen B, Yates P, Avision D. Neuropsychological syndromes in presenile dementia due to cerebral atrophy. J Neurol Neurosurg Psychyatry 1986, 49: 163-174.

19. Nespoulous L, Lecours AR, Lafond D, Lemay A, Puel M, Joanette Y, Cot F, Rascol A. Protocole MontrealToulose d'examen linguistique de l'aphasie. MT-86. Module standard initial: M1 bêta. Montreal: Laboratoire Theophile-Aajouanine, Centre de Recherche du Centre hospitalier Côte -des-Neiges.

20. Peffa Casanova J. Nomalidad, semiologia y patologia neuropsicologicas. Barcelona: Masson, 1991.

21. Osterrieth PA. Le test de copie d'une figure complexe. Arch Psychol 1945, 30: 205-353.

22. Seron X. La neuropsychologie cognitive. Paris: Presses Univ France, 1993.

23. Shallice T. Specific impairments of planning. Phylosoph Trans R Soc London 1982, 298: 199-209.

24. Ska B. Fonctions visuospatiales et praxiques dans la démence et syndromes démentiels: approche neuropsychologique, Paris: Masson \& Edissen, 1991, p 189-202.

25. Ska B, Desilets H, Joanette Y. Performances visuo-constructives et vieillissement. Psychol Belg 1986, 26: 125-145.

26. Ska B, Joanette Y, Poissant A, Béland R, Lecours AR. Language disorders in dementia of the Alzheimer type: contrastive pattems from a multiple single case study. Abstracts of the Academy of Aphasia 28th Annual Meeting Baltimore (USA),21-23 october, 1990.

27. Ska B, Poissant A, Joanette Y. Line orientation judgement in normal elderly and subjects with aphasia (28th Annual Meeting Baltimore (USA) 1990, October, p 21-23: dementia of Alzheimer's type). J Clin Exp Neuropsychol 1990, 12: 695-702.

28. Stroop JR. Studies of interference in serial verbal reaction. J Exp Psychol 1935, 18: 643-662.

29. Stuss DT, Benson F. The frontal lobes. New York: Raven Press, 1986.

30. Valdois $\mathbf{S}$, Joanette $\mathbf{Y}$. Hétérogéneité du déclin cognitif associé au vieillissement normal. In Habib $\mathbf{M}$, Joanette Y, Puel M. Démences et syndromes démentiels: approche neuropsychologique. Paris: Masson \& Edissen, 1991, p 135-144.

31. Vanier M, Gauthier L, Pépin EP, Dobouloz CJ, Gagnon R, Joanette Y. Evolution of left visuospatial neglect: norms and discrimination power of two tests. Neuropsychology 1990, 4: 87-96.

32. Wechsler D. Manuel de l'échelle clinique de mémoire de Wechsler. Paris: Ed Centre Psychol Appl, 1969. 
CORRIGENDA. O Editor recebeu carta do Professor Yves Joanette fazendo notar que, em respeito a cada um dos colaboradores e por uma questão de equidade, a ordem dos autores seja mudada em dois artigos de que $€$ o pesquisador responsável; faz notar também que os artigos parecem ter redação difícil de compreender e contêm expressōes não habitualmente utilizadas em neurologia do comportamento. Esses artigos são os seguintes: Brito-Marques PR, Joanette Y, Poissant A, Ska B. Avaliação neuropsicologica adequada ds demências [Optimal neuropsychological evaluation of dementias]. Arq Neuropsiquiatr 1995;53(1):147-152; Brito-Marques PR, Joanette Y, Ska B, Poissant $A$, Melaçon L, Giroux F. Heterogeneidade cognitiva na doença de Alzheimer [Cognitive heterogeneity in Alzheimer's disease]. Arq Neuropsiquiatr 1995;53(3-A):451-454.

Em relação ao primeiro aspecto e dentro das normas de publicações cientificas (ver International Committee of Medical Joumal Editors. Uniform requirements for manuscripts submitted 10 biomedical journals. $N$ Engl J Med 1991;324:424-428) o Editor considera justo o ponto de vista do Professor Yves Joanette e adota a ordem de autores por ele sugerida como chefe-de-escola. $O$ Editor deu ciência do fato ao Dr. Paulo Roberto de Brito Marques que aceitou a resolução. Assim, esses artigos passam a ser catalogados como segue:

- Joanette Y, Poissant A, Ska B, Brito-Marques PR. Avaliaçäo neuropsicológica adequada ds demências [Optlmal neuropsychological evaluation of dementias]. Arq Neuropsiquiatr 1995;53(1):147-152;

- Joanette Y, Ska B, Poissant B, Melanfon L, Gïroux F, Brito-Marques PR. Heterogeneidade cognitiva na doença de Alzheimer [Cognitive heterogeneity in Alzheimer's disease]. Arq Neuropsiquiatr 1995;53(3-A):451-454.

Em relação ao segundo aspecto e de acordo com as mesmas normas lembradas no parágrafo anterior, o Editor convidou o Professor Yves Joanette a escrever um editorial em que sejam salientadas as contribuiçōes dele e de sua escola a proposito do tema, com o qual as dificuldades e imprecisóes serão naturalmente dirimidas. O Editor espera do Professor Yves Joanette a gentileza de aceitar o convite. Esse editorial seguramente representará importante contribuiçăo ao periódico e aos seus leitores, em particular aos especialistas em neurologia do comportamento. $O$ Editor deixa registrado que, o editoral será publicado assim que recebido. ANTONIO SPINA-FRANÇA, Editor. 